\section{Nuclear Medicine Radiation Risks}

TO THE EDITOR: In recent times, there has been concern about the harmful effects of radiation from diagnostic procedures such as CT. The BEIR VII report (1) invoked data, largely but not entirely from atomic bomb survivors, to support the linear-nothreshold model of radiation-induced cancer down to the levels that are relevant to such procedures. Brenner and Hall (2) elaborated with estimates relating to the risk of CT. Presumably, these principles are also applicable to both diagnostic and therapeutic nuclear medicine.

Two recent articles in The Journal of Nuclear Medicine, by Boreham and Dolling (3) and by Stabin (4) express contrary views, questioning the linear-no-threshold model. Boreham and Dolling (3) even suggest that low levels of radiation are beneficial. Evidently, there is a controversy. I am not writing to take sides, although I must add that I find Stabin's (4) statement, "the benefits of the study...always substantially outweigh [the] risks," simplistic. In any case, the issues are important for nuclear medicine, having a bearing on selectivity for procedures, radiopharmaceutical doses used, and the information regarding risk that is given to patients and subjects in the contexts of both clinical service and research.

I hope that the SNM and The Journal of Nuclear Medicine will foster evenhanded dialog on this subject.

\section{REFERENCES}

1. National Research Council of the National Academies. Health Risks from Exposure to Low Levels of Ionizing Radiation: BEIR VII, Phase 2. Washington, DC: The National Academies Press; 2006.

2. Brenner DJ, Hall EJ. Computed tomography: an increasing source of radiation exposure. N Engl J Med. 2007;357:2277-2284.

3. Boreham DR, Dolling J-A. Risk associated with therapeutic ${ }^{131} \mathrm{I}$ radiation exposure. J Nucl Med. 2008;49:691-693.

4. Stabin MG. Radiopharmaceuticals for nuclear cardiology: radiation dosimetry, uncertainties, and risk. J Nucl Med. 2008;49:1555-1563.

\section{Herbert A. Klein Veterans Affairs Pittsburgh Healthcare System Pittsburgh, Pennsylvania}

DOI: 10.2967/jnumed.108.058099

REPLY: All reasonable measures of course should be taken to minimize patient exposure without compromising the clinical value of any medical procedure. We know, however, that significant benefits are afforded to individual subjects who are exposed to radiation in the course of diagnostic medical procedures and that the associated risks are small, even if we accept the tenets of the linearno-threshold model. Pat Zanzonico has now presented us with numeric estimates of the benefits of several medical studies. These data were presented at the 2007 ASNC meeting, and are currently posted at a Web site of the Health Physics Society (1). The complete analysis is to be published soon.

COPYRIGHT @ 2009 by the Society of Nuclear Medicine, Inc.

\section{REFERENCE}

1. Zanzonico P. Benefits of Medical Radiation Exposures. Health Physics Society Web site. Available at: http://hps.org/hpspublications/articles/Benefitsofmedradexposures. html. Accessed January 7, 2009.

Michael G. Stabin Vanderbilt University Nashville, Tennessee

DOI: 10.2967/jnumed.108.058222

\section{Characterization of Hilar and Mediastinal Foci on ${ }^{18}$ F-FDG PET: New Variables}

TO THE EDITOR: The article by Karam et al. (1) on the features of ${ }^{18}$ F-FDG-concentrating bilateral hilar foci associated with benign or malignant pathologies draws the reader's attention to an important and relevant issue of current PET practice in oncology. The need to develop a logical decision-making algorithm in this confounding situation and educate the interpreting physicians about such an approach to eliminate errors can hardly be overemphasized. I would like to congratulate the authors for their well-done analysis that attempts a solution to this common diagnostic dilemma. Despite significant recent progress in PET technology (e.g., emergence of fusion PET/CT and novel quantitative approaches), accurate characterization of ${ }^{18} \mathrm{~F}-\mathrm{FDG}$-concentrating mediastinal and hilar nodes continues to pose a major diagnostic challenge worldwide. This challenge also has geographic relevance and is of major concern in Asian countries, including India, where tuberculosis has a high prevalence, and hence, the possibility of encountering falsepositive ${ }^{18}$ F-FDG PET lesions is higher than in the West. Tuberculous lesions can demonstrate variable ${ }^{18} \mathrm{~F}-\mathrm{FDG}$ uptake as determined by inflammatory activity $(2-5) .{ }^{18} \mathrm{~F}$-FDG PET is being investigated for its potential in the assessment of disease activity in a wide array of infectious and aseptic inflammatory conditions $(5,6)$. Hence, scientific analyses of the patient data of the past 2 decades are much needed. Analysis of data that can give important clues about the diagnosis will be of help in devising an optimal approach, ultimately obviating invasive biopsies and reducing patient anxiety.

As the authors indicate (1), their study probably represents the first attempt to scientifically correlate multiple PET variables with ultimate outcome. The results of their retrospective analysis reaffirm certain traditional notions about the significance of variables such as symmetry, maximum standardized uptake value, node size on CT, and stability of ${ }^{18} \mathrm{~F}$-FDG uptake during the course of the disease-variables that are being followed in several PET centers in various countries. All these characteristics are important and performed as expected in determining the nature of the lesions in the study of Karam et al. In addition, the results underscore the importance of the absence or presence of ${ }^{18} \mathrm{~F}-\mathrm{FDG}$-avid foci in nonhilar mediastinal nodes, a variable (which they term the "purity" of the lesion) that was found to be an independent determinant after multivariate analysis. Seventy-nine percent of impure scans, versus $18 \%$ of pure scans, represented malignancy in the examined population. Also, the significant dependence of the nature of the 